\title{
Virulence of an emerging pathogenic lineage of Vibrio nigripulchritudo is dependent on two plasmids
}

\author{
Frédérique Le Roux ${ }^{1,2,{ }^{*}}$, Yannick Labreuche ${ }^{3}$, Brigid M. Davis ${ }^{2}$, Naeem Iqbal $^{4,5}$, Sophie Mangenot ${ }^{6}$, \\ Cyrille Goarant ${ }^{7}$, Didier Mazel ${ }^{4,5}$, Matthew K. Waldor ${ }^{2,8}$
}

${ }^{1}$ Laboratoire de Génétique et Pathologie, IFREMER, 3790 La Tremblade, France.

${ }^{2}$ Channing Laboratory/Brigham and Women's Hospital and Harvard Medical School, 181 Longwood Ave, Boston, MA 02115, USA.

${ }^{3}$ Département Lagons, Ecosystèmes et Aquaculture Durable en Nouvelle-Calédonie, IFREMER, BP 2059, 98846 Nouméa Cedex, New Caledonia.

${ }^{4}$ Institut Pasteur, Unité Plasticité du Génome Bactérien, Département Génomes et Génétique, F75015 Paris, France.

${ }^{5}$ CNRS, URA2171, F-75015 Paris, France.

${ }^{6}$ CEA, Institut de Génomique, Genoscope \& CNRS UMR8030 Laboratoire de Génomique Comparative, Evry, France.

${ }^{7}$ Institut Pasteur, Nouméa, New Caledonia.

${ }^{8}$ HHMI, Boston, MA 02115, USA.

*: Corresponding author : Frédérique Le Roux, email address : fleroux@ifremer.fr

\begin{abstract}
:
Vibrioses are the predominant bacterial infections in marine shrimp farms. Vibrio nigripulchritudo is an emerging pathogen of the cultured shrimp Litopenaeus stylirostris in New Caledonia and other regions in the Indo-Pacific. The molecular determinants of $V$. nigripulchritudo pathogenicity are unknown; however, molecular epidemiological studies have revealed that recent pathogenic $V$. nigripulchritudo isolates from New Caledonia all cluster into a monophyletic clade and contain a small plasmid, pB1067. Here, we report that a large plasmid, pA1066 (247 kb), can also serve as a marker for virulent $V$. nigripulchritudo, and that an ancestral version of this plasmid was likely acquired prior to other virulence-linked markers. Additionally, we demonstrate that pA1066 is critical for the full virulence of $V$. nigripulchritudo in several newly developed experimental models of infection. Plasmid pB1067 also contributes to virulence; only strains containing both plasmids induced the highest level of shrimp mortality. Thus, it appears that these plasmids, which are absent from non-pathogenic isolates, may be driving forces, as well as markers, for the emergence of a pathogenic lineage of $V$. nigripulchritudo.
\end{abstract}




\section{Introduction}

The family Vibrionaceae is comprised of a diverse group of organisms that reside within aquatic, mostly marine environments (Thompson et al., 2004). It encompasses the ancient and well-studied human pathogen, $V$. cholerae, as well as some less thoroughly characterized human pathogens, including $V$. parahaemolyticus and $V$. vulnificus. Perhaps less widely recognized are the consequences of vibrio infections in non-human species. Vibrios have been found to be pathogens of fish ( $V$. anguillarum), coral $(V$. shiloi), shellfish ( $V$. splendidus), and shrimp ( $V$. harveyi, $V$. penaeicida and $V$. nigripulchritudo), and infections with these organisms have profound environmental and economic consequences (Goarant et al., 2004; Rosenberg and Falkovitz, 2004; Lopez and Crosa, 2007; Le Roux et al., 2009; Austin, 2010).

Vibrioses are the predominant bacterial infections in marine shrimp culture systems (Park et al., 1994; Ninawe and Selvin, 2009). In New Caledonia, where the cultivated penaeid shrimp Litopenaeus stylirostris is the second largest export, the industry has been affected since 1993 by a cold season vibriosis caused by Vibrio penaeicida (Syndrome 93; Goarant et al., 1999) that has largely prevented cultivation during this season. Additionally, since 1997, outbreaks during the summer period ("Summer Syndrome") have been caused by V. nigripulchritudo (Goarant et al., 2006b); the increasing prevalence of these epizootics threatens the viability of shrimp farming in this area. Mass mortalities of other peneids (Marsupenaeus japonicus and Penaeus monodon) due to infection with $V$. nigripulchritudo have also been observed in Japan (Sakai et al., 2007) and in Madagascar (E. Chungue, pers.com.), suggesting that this organism potentially affects vast regions in the Indo-Pacific. Infections are characterized by an acute systemic vibriosis; however, little is known about the prevalence or diversity of pathogenic isolates of this organism or about the molecular basis of the disease that it causes.

In previous studies, $V$. nigripulchritudo isolated from a variety of sites in New Caledonia over an 8-year period have been characterized genetically and in an experimental model of infection (Goarant et al., 2006b; Goarant et al., 2006a). Multi Locus Sequence Typing (MLST) revealed that all isolates from shrimp affected by Summer Syndrome, regardless of the year or site of isolation, clustered into a monophyletic clade. Studies of mortality after experimental infection suggested that pathogenicity is linked to this lineage. All isolates of the monophyletic cluster were demonstrated to be moderately pathogenic (20-80\% mortality; MP) or highly pathogenic (80-100\% mortality; HP) when injected into shrimp, while nonpathogenic isolates (NP, 0-20\% mortality) were genetically diverse. MLST data did not permit discrimination between HP and MP isolates.

To further understand the differences between pathogenic and non-pathogenic isolates of $V$. nigripulchritudo, subtractive suppression hybridization (SSH; Winstanley, 2002) was performed using representative HP (SFn1) and NP (SFn118) isolate (Reynaud et al., 2008). DNA fragments isolated using this approach were subsequently scored, using macroarrays, for their presence in HP, MP, and NP strains. This study led to the identification of 13 DNA fragments detectable in the HP isolates and absent from both NP and MP isolates. Of these, 10 corresponded to putative ORFs harbored by a $11.2 \mathrm{kbp}$ plasmid, designated pB1067 (previously named pSFn1), raising the possibility that this element contributed to the emergence of HP strains (Reynaud et al., 2008). The study also identified 3 chromosomederived fragments that were found in nearly all HP strains (that we have now localized to a genomic island of chromosome 2) and 55 fragments that were shared by HP and MP strains 
but absent from NP strains, any of which might encode factors that contribute to $V$. nigripulchritudo virulence. The subsequent and ongoing sequencing of the complete genome of the $V$. nigripulchritudo HP strain SFn1 (Genoscope, Evry, France) revealed that two of the 55 fragments, which contain sequences homologous to transposases, are encoded within a previously undetected large $(247.2 \mathrm{kbp})$ plasmid here named pA1066. This finding raised the possibility that loci within pA1066 might be important for the pathogenicity of $V$. nigripulchritudo.

In this study, we assessed the prevalence of pA1066 or related plasmids among pathogenic and nonpathogenic isolates and found that HP and MP strains always carry distinct forms of this replicon. The precise correlation between the presence of the HP form of pA1066, pB1067, and previously described HP-specific chromosomal loci suggests that these features are genetically linked. We also explored the contribution of the HP-specific plasmids to the virulence of these strains. By infecting shrimp with derivatives of an HP $V$. nigripulchritudo strain from which pA1066 and/or pB1067 have been lost we found that both plasmids are necessary for full virulence, particularly when using a bath challenge method of infection that mimics natural infection conditions. In addition, we found that supernatant toxicity is a characteristic of HP, but not MP, strains. HP strains release a large, heatsensitive factor into culture supernatants that is toxic upon injection into shrimp, and the production and/or activity of this factor is dependent upon the presence of pA1066.

\section{Results}

\section{The large plasmid pA1066 encodes a putative MARTX toxin and may be conjugative}

Genome sequencing of $V$. nigripulchritudo strain SFn1 is in progress at the Genoscope (Evry, France). Although annotation of this genome is not yet completed, assembly of contigs revealed that SFn1 has the largest vibrio genome described to date, confirming observations previously made (Okada et al., 2005). It consists of two circular chromosomes of 4,062 (chromosome 1) and 2,214 (chromosome 2) kbp and two plasmids of 11.7 (pB1067) and 247.2 (pA1066) kbp, with an average percent $\mathrm{G}+\mathrm{C}$ content of 45.9, 45.5, 45.7 and 45.3\%, respectively. The plasmid pB1067 (accession number: NC_010733), which was previously described (Reynaud et al., 2008), contains 10 open reading frames (ORFs) and encodes putative partitioning protein, conjugal transfer repressor, peptidase $\mathrm{S} 49$, and 7 conserved hypothetical proteins. Identification of the large plasmid, pA1066, in the SFn1 genome was unexpected, since this replicon had not been previously detected.

A total of 179 ORFs were predicted from the pA1066 plasmid sequence (Figure 1). Nearly half of the pA1066 genes code for proteins of unknown function. Like many large plasmids, pA1066 appears to have a modular architecture, which is a likely consequence of evolutionary mosaicism (Fernandez-Lopez et al., 2006). Three notable putative functional modules include: a replication/segregation module (Figure 1 in supplementary data), a MARTX (multifunctional autoprocessing repeats in toxin) toxin cluster (Figure $2 \mathrm{~A}$ in supplementary data) and a cluster that appears to encode conjugation machinery (Figure 3 in supplementary data). The potential roles of additional genes with characterized homologs are annotated in the complete plasmid sequence (accession number: FP89324).

The putative Rep protein (VIBNI_0003) bears very limited homology to known proteins. However, it contains 3 short stretches of amino acids (6-15 residues) that are identical to 
sequences present in $\mathrm{RctB}$, the $V$. cholerae Chr2 replication initiator. Furthermore, VIBNI_0003 is flanked by a region that exhibits similar features found in V. cholerae oriC2, including a DnaA box, 18 repeat sequences, and an AT rich segment (Figure 1 in supplementary data), all of which are important for RctB-based replication in $V$. cholerae (Egan and Waldor, 2003). These observations, coupled with the proximity of parA/parB homologs (VIBNI_001 and _002) which are often found near origins of replication (Bignell and Thomas, 2001), suggest that this region might comprise the replicative origin of this plasmid. In support of this hypothesis, we found that a functional replicon was generated by

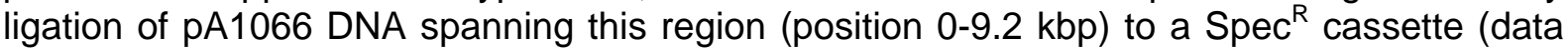
not shown). Notably, this plasmid replicated in $V$. cholerae but not in $E$. coli, as was also observed for some rctB-dependent replicons (Egan and Waldor, 2003).

A six-gene cluster $(211-232.9 \mathrm{kbp})$ in pA1066 that encodes a putative toxin (RtxA, VIBNI_0210), a putative acyltransferase (RtxC, VIBNI_0211), an uncharacterized protein (RtxH, VIBNI_0212), and a putative type I secretion system (RtxBDE, VIBNI_0213 to 15) shows an organization similar to MARTX toxin clusters (Figure 2A in supplementary data). These clusters, which have been characterized primarily in $V$. cholerae and $V$. vulnificus but have also been found in numerous other organisms enable secretion of large, typically multifunctional, protein exotoxins, which are encoded by $r t x A$ (reviewed by Satchell, 2007). Close to the rtxA gene we identified a pseudogene homologous to recombinase, suggesting that this cluster was acquired via horizontal gene transfer.

As in $V$. cholerae and $V$. vulnificus, the putative $V$. nigripulchritudo RtxA is extremely large $(4,990$ amino acids; $536 \mathrm{kDa})$ and contains numerous modules (generously annotated by $\mathrm{Dr}$. Karla Satchell; Figure 2B in supplementary data). It is predicted to contain the 3 domains found in all MARTX: large repeat regions at the $\mathrm{N}$ - and $\mathrm{C}$-termini, and a cysteine protease domain that is essential for the autoprocessing and toxicity of MARTX in $V$. cholerae (Sheahan et al., 2007). In addition, it contains putative effector domains that have been identified in a subset of other MARTX toxins. These include a homolog of the Rho GTPase domain that has been associated with the "cell rounding" effect caused by MARTX $\mathrm{Vc}_{\mathrm{v}}$ (Sheahan and Satchell, 2007), an ExoY adenylate cyclase domain, and two domains with unknown function, DUF1 and DUF3. The closest homologs for each domain are found within distinct organisms, suggesting that the pA1066 MARTX toxin, like other members of this family, is a mosaic of independently acquired effector domains (Satchell, 2007).

The pA1066 sequence also includes a gene cluster (114.7-140.7 kbp; VIBNI_111 to 127) that may impart mobility to the plasmid. This cluster includes homolog of the core proteins of a Type IV secretion system, the coupling protein TraD and a relaxase-helicase Tral (Figure 3 in supplementary data) (Lawley et al., 2003). The presence of these genes suggests that pA1066 may be a conjugative plasmid; however, since we have been unable to generate a marked version of this replicon (discussed below), it has not been possible to assess this experimentally. The GC \% average of this region (54\%) is clearly different from the whole plasmid $(45.3 \%)$ and the region is flanked by two pseudogenes - a transposase and a helicase - suggesting that these sequences evolved separately from the remainder of the plasmid and were acquired via horizontal gene transfer. 
HP and MP strains contain distinct forms of pA1066

The finding that 2 of $55 \mathrm{HP} / \mathrm{MP}$ specific fragments identified by $\mathrm{SSH}$ are encoded within pA1066 suggested that this large plasmid might be linked to virulence. Therefore we undertook a more systematic evaluation of the presence of this plasmid within our V. nigripulchritudo collection (Goarant et al., 2006b). Several attempts to detect pA1066 by pulse field gel analysis failed, apparently due to DNA degradation, which tends to be more extensive in DNA samples from $V$. nigripulchritudo than in those from other Vibrio species (data not shown). Consequently, we amplified at 1500 bp fragment of the putative rep gene from this collection of isolates. This region was successfully amplified from all HP and MP strains tested and from 3 of 15 NP strains. Sequence analysis revealed that this region is identical within all HP and MP strains, while NP strains contain several distinct sequence variants. Thus, phylogenetic analysis of pA1066 rep (Figure 2) is fully congruent with the previous phylogenetic analyses based on MLST data, in that both led to the grouping of MP/HP strains in a monophyletic group, whereas NP strains were found to be polyphyletic.

Since strains within this monophyletic cluster have distinct phenotypes in the experimental model of infection (i.e., some HP, some MP), we explored whether variability in regions of pA1066 other than its origin could be detected among these strains, which could potentially account for the range of phenotypes. For this analysis, we attempted to amplify 10 regions of $2 \mathrm{kbp}$ that are distributed at $25 \mathrm{kbp}$ intervals around pA1066 from each strain (Figure $1,3^{\text {rd }}$ circle). Each HP strain tested yielded a product of the expected size for 10/10 primers sets, suggesting that a pA1066-like plasmid (named type HP) is present in all these HP isolates (Table 1). In contrast, all MP isolates tested yielded a product of the expected size for only $8 / 10$ primers sets (including MARTX). No product was obtained from MP strains for 2 primer sets (PCR 7, 8). Thus, it appears that MP strains consistently carry a different form of pA1066 (named pA1066 type MP) than the one present in HP strains. The proteins whose presence may be restricted to HP isolates include a putative siderophore $A B C$ transporter and a putative metalloprotease, both of which are linked to virulence in other pathogenic vibrios (Le Roux et al., 2007; Lopez and Crosa, 2007). Alternatively, sequence polymorphisms may account for their failure to be amplified. Only 4 of $15 \mathrm{NP}$ isolates yielded any PCR products in these analyses, and no NP strain yielded more than 4 products (Table 1). This result is consistent with our previous observation that pA1066 rep can rarely be amplified from NP strains, and suggests that any pA1066-like plasmid present in an NP isolate is only distantly related. Collectively, these results suggest that the presence of an HP-type pA1066 plasmid, like the previously demonstrated presence of pB1067, is a reliable marker of high pathogenicity in New Caledonian V. nigripulchritudo. Furthermore, the identity of the sequence of the rep gene in MP- and HP-type pA1066 plasmids suggests that these plasmids are derived from a common ancestor that has subsequently differentiated into the MP and HP types.

\section{Disruption and curing of HP-linked plasmids in V. nigripulchritudo}

Given the correlation between the presence of pA1066, pB1067, and high pathogenicity, we wanted to assess the importance of either or both of these plasmids for $V$. nigripulchritudo virulence. We therefore attempted to disrupt potential virulence-linked ORFs in each replicon, by introducing a suicide vector, derived from the oriR6K-dependent vector pSW25T (Demarre et al., 2005), that would integrate within these loci. In addition, we attempted to cure each plasmid from the HP strain SFn1 by introducing an incompatible and selectable replicon. All of these genetic manipulations relied upon conjugation to introduce sequences into SFn1, as successful protocols for transformation of $V$. nigripulchritudo have not yet been established. Using this approach, we managed to disrupt numerous genes within pB1067 by insertional mutagenesis (Tables 2 and 3 and data not shown); however, strains in which a suicide vector had integrated within pA1066 could never be obtained. Subsequently, during 
characterization of the pB1067 mutants, we noted that all of these strains no longer contained pA1066 (data not shown). Similarly, when pSW110, which consists of the pB1067 origin of replication (see methods) fused to pSW25T was introduced into SFn1 in order to cure it of pB1067, pA1066 was also lost from the majority (9 of 10) of strains (data not shown). Collectively, these results suggest that the presence of pSW25T sequences, either integrated into another replicon (e.g., as in insertional disruption of pB1067) or within an independent replicon (e.g., as in pSW110), interferes with replication and/or maintenance of pA1066. The mechanism underlying the incompatibility of these sequences has not yet been determined. However, we found that conjugative transfer to SFn1 of a P15A-dependent plasmid that confers spectinomycin resistance yielded transconjugants that contained both pB1067 and pA1066 (not shown), suggesting that neither the resistance cassette nor the process of conjugal DNA transfer are likely to interfere with maintenance of pA1066. It seems therefore most likely that the R6K origin of replication in pSW25T interferes, in an unknown fashion, with pA1066, and that this accounts for the inability to introduce mutations within pA1066 noted above.

Fortunately, by removing antibiotic selection from the single SFn1 derivative that maintained pA1066 in the presence of pSW110, we were able to isolate a strain that contained only pA1066 (referred to as VN157). Subsequent transfer of a pB1067::pSW120 cointegrate into VN157 resulted in loss of pA1066, indicating that pA1066 within VN157 has not become routinely compatible with pSW25T and suggesting that this plasmid maintains its natural characteristics. The wild-type SFn1, VN157, the numerous strains containing only derivatives of pB1067, and VN110 (cured of both plasmids; contains only the pB1067 origin of replication fused to pSW25T) all displayed similar kinetics of growth and final absorbance at $600 \mathrm{~nm}$ in different media, indicating that plasmid curing did not have deleterious effects upon growth (not shown). The virulence of these strains was therefore assessed in experimental models of $V$. nigripulchritudo infection.

\section{Experimental challenge demonstrates that both plasmids are necessary for virulence}

The previous study of $V$. nigripulchritudo pathogenicity that yielded the distinction between HP, MP, and NP strains utilized an experimental infection model in which bacteria are intramuscularly injected into healthy juvenile shrimp (L. stylirostris). While this assay is fairly convenient and yields reproducible results, it is not particularly analogous to the natural route of infection. We therefore assayed the virulence of our mutants using 3 experimental models: shrimp intramuscularly injected with bacteria, shrimp transiently immersed into bacteria-contaminated waters, and shrimp intramuscularly injected with bacterial culture supernatants. Collectively, these experiments revealed that pA1066 and pB1067 are both required for full pathogenicity of $V$. nigripulchritudo, although their results differ as to whether the effects of these plasmids are independent or interdependent. In addition, they indicate that at least some of the virulence of $V$. nigripulchritudo may be due to pA1066-dependent release of a toxic compound.

As previously reported, intramuscular injection of the HP strain SFn1 into shrimp results in the death of $>80 \%$ of shrimp (typically 100\%) within 24 hours (Figure 3A; (Goarant et al., 2006b)). In contrast, shrimp injected with VN68, VN120, and VN110, which lack pA1066 or both plasmids, displayed a maximum mortality of $20 \%$ even 3 days post-injection. This result suggests that pA1066 is crucial for the high pathogenicity phenotype of strain SFn1. Injection of VN157, which contains pA1066 but lacks pB1067, resulted in an intermediate phenotype. Approximately $70 \%$ of shrimp died, but the majority did not succumb to the infection until at least 2 days post-injection. Thus, pB1067 is not pivotal for virulence, but it does augment virulence in this model. 
To more accurately model the natural process by which vibriosis develops in shrimp, we utilized an immersion model of infection (Saulnier et al., 2000). Shrimp were placed in filtered seawater contaminated with $V$. nigripulchritudo only transiently $(2 \mathrm{hr})$, then transferred to clean water for the duration of the experiment. Under these assay conditions, only $V$. nigripulchritudo containing both pA1066 and pB1067 induced significant mortality ( $>80 \%$ by 48 hours; Figure 3B). Strains containing 1 or no plasmid induced $<20 \%$ mortality, even several days after infection. For the strains containing no plasmid this low mortality may result from a reduced ability to infect/colonize the host shrimp, as enumeration of $V$. nigripulchritudo in shrimp hemolymph $24 \mathrm{hrs}$ after exposure revealed that their abundance was several orders of magnitude lower than that of wt SFn1 (SFn1 and VN157: \#10 CFU/mL; VN68 and VN120: \#10 ${ }^{3} \mathrm{CFU} / \mathrm{mL}$; VN110: $0 \mathrm{CFU} / \mathrm{mL}$ ). However, the bacteriaemia of wt SFn1 and VN157 bacteria was equivalent, suggesting that colonization by $V$. nigripulchritudo is not sufficient to induce mortality in L. stylirostris; additional, presumably pB1067-dependent factors, must also be necessary.

Finally, we assessed whether products released by the various strains of $V$. nigripulchritudo into their surroundings were detrimental to L. stylirostris. In vivo injection of culture supernatants, extracellular products (ECP), or purified metalloproteases is a classic assay of bacterial exo-toxicity (Goarant et al., 2000; Labreuche et al., 2006; Le Roux et al., 2007); We therefore tested the exotoxicity of a diverse panel of $V$. nigripulchritudo isolates, including strains previously classified as HP $(n=4), M P(n=2)$, and NP $(n=2)$, in addition to testing supernatants derived from our set of SFn1 derivatives. Strikingly, one day post-injection, $10 \%$ and $0 \%$ mortality was observed with the MP and NP supernatants, respectively, whereas $90-100 \%$ cumulative mortality resulted from injection of supernatants prepared from all 4 HP strains (Figure 4). Thus, exotoxicity appears to be an HP-specific phenotype, and may be useful for future classification of new $V$. nigripulchritudo isolates. Among SFn1derived strains, only VN157 demonstrated notable exotoxicity. Nearly $100 \%$ of shrimp injected with supernatant from this strain died within $24 \mathrm{hrs}$, as was observed using supernatant from wt SFn1. In contrast, strains containing only a pB1067-derived plasmid induced mortality in $0-20 \%$ of shrimp. These data indicate that pA1066 is necessary and sufficient to induce supernatant toxicity.

To gain insight into the nature of the toxic factor, SFn1 supernatants were either heat treated at $85^{\circ} \mathrm{C}$ for $15 \mathrm{~min}$ or size-fractionated by filtration (cut-off of 10 or $50 \mathrm{kDa}$ ) prior to shrimp injection. Only untreated crude extracts induced mortality, suggesting that one or more proteinaceous compounds with a molecular weight $>50 \mathrm{kD}$ might be implicated in toxicity (not shown). SFn1 supernatant treated with protease inhibitors (10 mM EDTA or $10 \mathrm{mM}$ 1, 10phenanthroline) had an effect on shrimp equal to that of untreated supernatant, suggesting that metalloprotease-like enzymes, such as those produced by $V$. splendidus (Le Roux et al., 2007) and V. tubiashi (Hasegawa et al., 2008) that induce damage in Pacific oysters, are not pivotal for $V$. nigripuchritudo pathogenicity. Finally, culture supernatants were analyzed using SDS-PAGE, in an attempt to identify toxin candidates; however, the complexity of these supernatants prevented identification of virulence-linked differences (data not shown). 


\section{Discussion}

The virulence of $V$. nigripulchritudo towards the shrimp $L$. stylirostris has been previously correlated with the presence of a small plasmid, pB1067, and a few chromosomal markers (Reynaud et al., 2008). In this study, we report that a second plasmid, pA1066, identified during genomic sequencing of a HP $V$. nigripulchritudo isolate, can also serve as a marker of the HP phenotype. A variant of this plasmid is present in MP strains (pA1066 type MP), suggesting that a precursor of pA1066 was acquired by the common ancestor of MP and HP strains; however, these plasmids have diverged, as they can readily be distinguished by PCR analyses. Notably, we demonstrate that pA1066 is critical for the full virulence of HP $V$. nigripulchritudo in several experimental models of infection. Plasmid pB1067 also contributes to virulence; in most models, only strains containing both plasmids induced the highest level of shrimp mortality. Thus, the emergence of strains of $V$. nigripulchritudo that are highly pathogenic for $L$. stylirostris appears to depend on the presence of two plasmids, pB1067 and a distinct form of pA1066, and is correlated with several previously described chromosomal polymorphisms.

Understanding the nature of pA1066's contribution to $V$. nigripulchritudo virulence should provide significant insight into the disease process induced in shrimp by this bacterium. However, analysis of this question has been significantly complicated by the difficulty of performing genetic manipulations on this plasmid. We have attempted to disrupt numerous genes within this replicon; however, all suicide vectors that we have tested appear to be incompatible with pA1066, as no co-integrants could be obtained. Consequently, it has not been possible to disrupt candidate virulence genes or the many genes of unknown function within this replicon, to address the significance of the encoded proteins in $V$. nigripulchritudo pathogenicity, or to assess whether pA1066 is a conjugative plasmid. This apparent incompatibility also complicated our analysis of the role of the co-resident plasmid, pB1067, as suicide vector integration within pB1067 typically results in the loss of pA1066. In future studies, we will explore whether non-R6K-based vectors or chitin-based natural transformation may enable genetic manipulation of pA1066 (Meibom et al., 2005; Gulig et al., 2009). Once mutations can be generated, it will be possible to investigate the function of numerous features of this large plasmid. For instance, we can explore if pA1066 is a selftransmissible conjugative plasmid. Plasmid pA1066 might also enable transmission of other, non-conjugative plasmids, such as pB1067, as has been observed for conjugative plasmids in V. vulnificus (Lee et al., 2008) and V. fisheri (Dunn et al., 2005). Transmission of both pA1066 and pB1067 could potentially promote development of new pathogenic strains. Conjugation/secretion systems have also been found to enable the secretion of virulence proteins directly into host cells (e.g., in Bordetella pertussis and Helicobacter pylori; (Christie and Vogel, 2000)); thus, it is also possible that these pA1066 genes directly contribute to virulence.

One gene cluster within pA1066 whose contribution to virulence clearly warrants investigation encodes a putative MARTX toxin and its transporter. Such toxins have been associated with virulence of $V$. cholerae (Lin et al., 1999), V. vulnificus (Liu et al., 2007) and $V$. anguillarum (Li et al., 2008), and they have been identified in several other pathogens as well (Satchell, 2007). As a secreted toxin, MARTX could be linked to the mortality that results from injecting shrimp with $V$. nigripulchritudo culture supernatants, particularly since the active factor appears to be a protein of $>50 \mathrm{kD}$. Arguing against this possibility is the fact that supernatants from MP strains, which also contain a MARTX gene cluster (within pA1066 type $\mathrm{MP}$ ), do not induce mortality. However, it is possible that protein expression or secretion differs between the HP and MP strains or that this toxin acts in concert with additional, HPspecific factors. The potential toxin domains encoded within the pA1066 MARTX cluster 
include a Rho GTPase inactivation domain (RID), an adenylate cyclase domain (ExoY), and two domains of unknown function. Rho GTPases have previously been shown to induce cell rounding; we used previously developed cell culture-based assays (Binesse et al., 2008) to determine whether supernatants from various $V$. nigripulchritudo strains also induced this effect. However, supernatants from HP, MP, and NP strains all induced a significant cytopathic effect, including cell rounding, perhaps due to the presence of abundant proteases within these supernatants (C. Delsert, pers.com). Consequently, it was not possible to ascertain whether MARTX-linked changes in cell morphology occurred, as such changes could easily be masked by changes induced by other factors produced by all strains.

In this study we supplemented our previously used model of virulence (intramuscular injection of bacteria) with two additionnal assays to evaluate the pathogencity of $V$. nigripulchritudo. The non-invasive immersion model mimics the natural infection process, but is quite laborious, while the injection of supernatants enables rapid and reproducible screening. The results from these assays were congruent in several aspects, particularly in demonstrating that pA1066 is required for virulence in all three models, and thus that pB1067, while present in the pathogenic isolates, is not sufficient for their virulence. However, the differences among them suggest that each assay may enable dissection of discrete aspects of the virulence process, for example concerning the role of pB1067. In the immersion model, only strains containing both pA1066 and pB1067 were virulent, while in bacterial injection model, SFn1, which contains both plasmids, was more virulent than a strain containing pA1066 alone. When supernatants were injected, toxicity was independent of the presence of pB1067. Collectively, these findings suggest that there may be interactions between factors encoded on the two plasmids. It is likely that differences between results obtained with the models reflect the different host-imposed barriers that are encountered by the bacteria. They may also indicate that there are multiple pathways by which the bacterium impairs shrimp viability.

MLST analyses have demonstrated that HP strains, along with MP strains, differ at several chromosomal loci from NP strains, and that they comprise a monophyletic clade that is evolutionarily quite distant from the NP isolates (Goarant et al., 2006a). Subsequent SSH analyses identified a small plasmid whose presence was limited to HP isolates, and several chromosomal loci whose presence largely correlated with the HP phenotype (Reynaud et al., 2008). Here, we have found that HP and MP strains both contain large plasmids that share a common origin, based on the sequence identity of their rep gene (Figure 2) and other loci (not shown). Related plasmids were only rarely detected among NP isolates, and they formed a distinct evolutionary unit, congruent with the MLST data. PCR analyses suggest that the pA1066 form HP plasmid may contain sequences that are absent from the MP form. Collectively, these results suggest that a pA1066 ancestor was the first of the 3 virulencelinked markers to be acquired. Subsequently, the current HP strains acquired pB1067, the HP-specific modification of pA1066, and the chromosomal polymorphisms; however, the relative order of these events cannot be determined, due to the absence of strains containing only a subset of these HP-specific features within our collection.

The absence of such intermediate forms suggests several interesting possibilities regarding the evolution of HP strains. First, such strains may have arisen relatively recently, which would be consistent with the lack of sequence polymorphisms in pA1066 among the various isolates. Additionally, the apparent temporal coupling of pB1067 acquisition and modifications of pA1066 and the chromosome suggests that these events may be interconnected, i.e. that changes in one locus prompted or provided a selective force for subsequent changes. Presumably the combination of changes that led to the emergence of the HP lineage has rendered it particularly suited to a niche or habitat. 
It remains to be seen whether chromosomal polymorphisms, like the $2 \mathrm{HP}$-specific plasmids, are required for full virulence; it is possible that they serve as markers of this lineage without directly contributing to pathogenicity. Still, it seems clear that the pathogenic phenotype is a feature of a very specific subset of strains with largely uniform genetic content. Comparisons of the complete genomes of several HP, MP and NP organisms, along with the development of new genetic tools for engineering of $V$. nigripulchritudo, will yield insight into the key features underlying virulence, and facilitate exploration of whether horizontal gene acquisitions have driven the differentiation of this virulent lineage.

\section{Materials and methods}

\subsection{Bacterial strains, plasmids and culture conditions}

E. coli strains DH5 $\alpha \lambda$ pir and $\beta 2163$ (Demarre et al., 2005) were used for cloning and conjugation, respectively. The $V$. nigripulchritudo unmodified isolates have been described previously (Goarant et al., 2006b). SFn1 derivatives and plasmids used in this study are described in Tables 2 and 3. V. nigripulchritudo strains were grown in $\mathrm{LB}+\mathrm{NaCl} 0.5 \mathrm{M}$, marine broth (MB) $1 \mathrm{X}$ or $5 \mathrm{X}$, or on marine agar (MA) at $30^{\circ} \mathrm{C}$. E. coli strains were grown in Luria-Bertani (LB) at $37^{\circ} \mathrm{C}$. Matings between $E$ coli and $V$. nigripulchritudo were performed at $30^{\circ} \mathrm{C}$ as described previously (Le Roux et al., 2007). Spectinomycin was used at $100 \mu \mathrm{g} / \mathrm{ml}$. Diaminopimelate (DAP) was supplemented when necessary to a final concentration of 0.3 $\mathrm{mM}$.

\section{Molecular biology techniques}

PCR was performed using Pfu Ultra DNA polymerase (Stratagene) or Hot Start DNA polymerase (Qiagen), according to the manufacturer's instructions. Primers are listed in supplementary data Table 1. Plasmid DNA was isolated using a spin miniprep kit (Qiagen).

\section{Plasmid construction}

A fragment of pB1067 that enables autonomous replication of a suicide vector (Le Roux et al., in prep) was amplified with primers pB2700f and pB3400r then cloned into BamH1 site of pSW25T, yielding pSW110. Nucleotides 2000-2530 of pB1067 were amplified with primers pB2000f and pB2530r. The resulting product was digested with $\mathrm{BamHI}$ and ligated to pSW25T, yielding pSW68. Nucleotides 28-480 of pB1067 were amplified with primers pB28f and pB480r, then similarly digested and ligated to yield pSW120. The results of all ligations were confirmed by plasmid sequencing using primers SW25seqS and AS.

\section{Strain construction}

Plasmids were conjugated from $\beta 2163$ into various $V$. nigripulchritudo strains. To generate strains VN110 and VN157, 10 Spec $^{R}$ exconjugants generated following transfer of pSW110 to SFn1 were restreaked, then scored by PCR or plasmid prep for the presence of pSW110, pA1066, and pB1067, using primers SW25seqS and SW25seqAS, pA1f and pA1r or pB28f and pB480r respectively. Of these $10 \mathrm{Spec}^{R}$ colonies, 9 lacked pA1066 and pB1067, and one of these was named VN110. The single $\mathrm{Spec}^{\mathrm{R}}$ isolates that from pB1067 but contained pA1066 was cultivated in LB/NaCl without antibiotic selection. After two subcultures $(1 / 50)$, a 
$\mathrm{Spec}^{\mathrm{S}}$ clone that had lost pSW110 but maintained pA1066 was identified. No loss of pA1066 from this strain (VN157) was observed following growth of additional subcultures.

Spec $^{R}$ exconjugants generated by transfer of pSW68 and pSW120 into SFn1 were scored, using PCR, for recombination of each plasmid with the appropriate site in pB1067 and for the presence of pA1066. All exconjugants were found to lack pA1066. Transfer of pSW68 yielded VN68, and transfer of pSW120 yielded VN120.

\section{Experimental challenge}

V. nigripulchritudo strains and mutants were cultured in Marine Broth for $18 \mathrm{~h}$ at $30{ }^{\circ} \mathrm{C}$ with constant shaking, yielding late exponential phase cultures. Bacterial concentrations in these cultures were determined by comparing their optical density at $600 \mathrm{~nm}$ to a previously established reference curve. Experimental infections in L. stylirostris shrimp were performed either by immersion or by intramuscular injection of bacterial cell suspensions or supernatants between the third and fourth abdominal segments.

For the injection challenge, 50 to 500 colony forming units (cfu) were intramuscularly injected into healthy shrimp kept in aquaria (2 replicate aquaria with 10 to 20 shrimp in each for each strain) filled with filtered seawater, aerated and held at $27^{\circ} \mathrm{C}$. Control shrimp were injected with an equal volume of sterile culture medium. Survival was monitored daily over a 3-day period, because preliminary trials demonstrated that no significant mortality occurred after this time.

Bacterial supernatants were prepared from bacterial cultures grown in MB $5 \mathrm{X}$ as described previously (Goarant et al., 2000). When indicated, crude supernatants were either denaturated by heat treatment at $85^{\circ} \mathrm{C}$ for $15 \mathrm{~min}$ or size-fractionated by filtration through Centricon YM-10 and YM-50 ultrafiltration units (Millipore, molecule weight cut-off, 10 and $50 \mathrm{kDa}$, respectively). Animals were injected with $100 \mu \mathrm{l}$ of each preparation or control medium. Experiments were conducted in duplicate (8 to 12 shrimp/aquarium). Toxicity was assessed after 24 hours.

Immersion challenges were performed in $100 \mathrm{~L}$ of filtered and aerated seawater containing $10^{5} \mathrm{CFU} / \mathrm{ml}$ of the considered vibrio strain (Saulnier et al., 2000). Following a 2 hour challenge, 10 to 20 individuals were transferred to $100 \mathrm{~L}$ tanks filled with filtered seawater, aerated and held at $27^{\circ} \mathrm{C}$. Control shrimp were treated as above except that bacterial cultures were not added during exposure periods. Three replicate tanks were used for each treatment. For each type of challenge, experiments were repeated twice. Two and $24 \mathrm{hrs}$ after infection, 4 shrimp from each experimental condition were randomly sampled. Hemolymph was collected using a 25-gauge needle containing an anticoagulant solution (2\% $\mathrm{NaCl}, 0.1 \mathrm{M}$ glucose, $30 \mathrm{mM}$ sodium citrate, $26 \mathrm{mM}$ citratic acid, $10 \mathrm{mM}$ EDTA) and serial dilutions were plated onto solid Marine agar with $2 \%$ glycerol $(\mathrm{w} / \mathrm{v})$ and incubated at $30^{\circ} \mathrm{C}$ for 72 h. Putative V. nigripulchritudo colonies were identified as black colonies.

\section{In silico analysis}

The annotation of pA1066 has been performed using the MaGe software (Magnifying Genome, (Vallenet et al., 2006)) (http://www.genoscope.cns.fr/agc/mage).

The rep sequences were aligned using Seaview and phylogenetic trees were built using Phylo-win program (Galtier et al., 1996) applied to Neighbour Joining method and Kimura's 2-parameter distances. Reliability of topologies was assessed by the bootstrap method with 1000 replicates. The plasmid map of pA1066 (Figure 1) was generated using the BioPython and GenomeDiagram (Pritchard et al., 2006) open software packages. 


\section{Acknowledgements}

The authors acknowledge Dr Karla Stachell, Dr Stephane Duigou and Evelyne Krin for annotation assistance; Dominique Ansquer, Marlène Vic, Laurane Pallandre and the zootechnical staff of the station Ifremer Nouvelle Calédonie for technical assistance. We wish to thank Dr Géraldine Van der Auwera for the plasmid map and Yann Boucher for fruitful discussions. Authors acknowledge support from the Genoscope and CEA for plasmid sequencing. DM acknowledges support from Institut Pasteur and CNRS. MKW acknowledges support from HHMI and NIH R37-AI42347

\section{References}

Austin, B. (2010) Vibrios as causal agents of zoonoses. Vet Microbiol 140: 310-317.

Bignell, C., and Thomas, C.M. (2001) The bacterial ParA-ParB partitioning proteins. J Biotechnol 91: 1-34.

Binesse, J., Delsert, C., Saulnier, D., Champomier-Verges, M.C., Zagorec, M., MunierLehmann, H. et al. (2008) Metalloprotease vsm is the major determinant of toxicity for extracellular products of Vibrio splendidus. Appl Environ Microbiol 74: 7108-7117.

Christie, P.J., and Vogel, J.P. (2000) Bacterial type IV secretion: conjugation systems adapted to deliver effector molecules to host cells. Trends Microbiol 8: 354-360.

Demarre, G., Guerout, A.M., Matsumoto-Mashimo, C., Rowe-Magnus, D.A., Marliere, P., and Mazel, D. (2005) A new family of mobilizable suicide plasmids based on broad host range R388 plasmid (IncW) and RP4 plasmid (IncPalpha) conjugative machineries and their cognate Escherichia coli host strains. Res Microbiol 156: 245-255.

Dunn, A.K., Martin, M.O., and Stabb, E.V. (2005) Characterization of pES213, a small mobilizable plasmid from Vibrio fischeri. Plasmid 54: 114-134.

Egan, E.S., and Waldor, M.K. (2003) Distinct replication requirements for the two Vibrio cholerae chromosomes. Cell 114: 521-530.

Fernandez-Lopez, R., Garcillan-Barcia, M.P., Revilla, C., Lazaro, M., Vielva, L., and de la Cruz, F. (2006) Dynamics of the IncW genetic backbone imply general trends in conjugative plasmid evolution. FEMS Microbiol Rev 30: 942-966.

Galtier, N., Gouy, M., and Gautier, C. (1996) SEAVIEW and PHYLO_WIN: two graphic tools for sequence alignment and molecular phylogeny. Comput Appl Biosci 12: 543-548.

Goarant, C., Merien, F., Berthe, F., Mermoud, I., and Perolat, P. (1999) Arbitrarily primed PCR to type Vibrio spp. pathogenic for shrimp. Appl Environ Microbiol 65: 1145-1151.

Goarant, C., Herlin, J., Ansquer, D., Brizard, R., and Marteau, A.L. (2004) Vibrio penaeicida and Syndrome 93 in New Caledonian prawn farming: review and perspectives. In C. Goarant, A. Herbland, Y. Harache, C. Mugnier (ed.), Styli 2003, Trente ans de crevetticulture en Nouvelle-Calédonie, Ifremer ed., Actes Colloq. (in French with English abstract). 203-209. Goarant, C., Herlin, J., Brizard, R., Marteau, A.L., Martin, C., and Martin, B. (2000) Toxic factors of Vibrio strains pathogenic to shrimp. Dis Aquat Organ 40: 101-107.

Goarant, C., Reynaud, Y., Ansquer, D., de Decker, S., Saulnier, D., and le Roux, F. (2006a) Molecular epidemiology of Vibrio nigripulchritudo, a pathogen of cultured penaeid shrimp (Litopenaeus stylirostris) in New Caledonia. Syst Appl Microbiol 29: 570-580.

Goarant, C., Ansquer, D., Herlin, J., Domalain, F., Imbert, F., and de Decker, S. (2006b) "Summer Syndrome" in Litopenaeus stylirostris in New Caledonia: Pathology and epidemiology of the etiological agent, Vibrio nigripulchritudo. Aquaculture 253: 105-113.

Gulig, P.A., Tucker, M.S., Thiaville, P.C., Joseph, J.L., and Brown, R.N. (2009) USER friendly cloning coupled with chitin-based natural transformation enables rapid mutagenesis of Vibrio vulnificus. Appl Environ Microbiol 75: 4936-4949. 
Hasegawa, H., Lind, E.J., Boin, M.A., and Hase, C.C. (2008) The extracellular metalloprotease of Vibrio tubiashii is a major virulence factor for pacific oyster (Crassostrea gigas) larvae. Appl Environ Microbiol 74: 4101-4110.

Labreuche, Y., Soudant, P., Goncalves, M., Lambert, C., and Nicolas, J.L. (2006) Effects of extracellular products from the pathogenic Vibrio aestuarianus strain $01 / 32$ on lethality and cellular immune responses of the oyster Crassostrea gigas. Dev Comp Immunol 30: 367379.

Lawley, T.D., Klimke, W.A., Gubbins, M.J., and Frost, L.S. (2003) F factor conjugation is a true type IV secretion system. FEMS Microbiol Lett 224: 1-15.

Le Roux, F., Binesse, J., Saulnier, D., and Mazel, D. (2007) Construction of a Vibrio splendidus mutant lacking the metalloprotease gene vsm by use of a novel counterselectable suicide vector. Appl Environ Microbiol 73: 777-784.

Le Roux, F., Zouine, M., Chakroun, N., Binesse, J., Saulnier, D., Bouchier, C. et al. (2009) Genome sequence of Vibrio splendidus: an abundant planctonic marine species with a large genotypic diversity. Environ Microbiol 11: 1959-1970.

Lee, C.T., Amaro, C., Wu, K.M., Valiente, E., Chang, Y.F., Tsai, S.F. et al. (2008) A common virulence plasmid in biotype 2 Vibrio vulnificus and its dissemination aided by a conjugal plasmid. J Bacteriol 190: 1638-1648.

Li, L., Rock, J.L., and Nelson, D.R. (2008) Identification and characterization of a repeat-intoxin gene cluster in Vibrio anguillarum. Infect Immun 76: 2620-2632.

Lin, W., Fullner, K.J., Clayton, R., Sexton, J.A., Rogers, M.B., Calia, K.E. et al. (1999) Identification of a vibrio cholerae RTX toxin gene cluster that is tightly linked to the cholera toxin prophage. Proc Natl Acad Sci U S A 96: 1071-1076.

Liu, M., Alice, A.F., Naka, H., and Crosa, J.H. (2007) The HlyU protein is a positive regulator of rtxA1, a gene responsible for cytotoxicity and virulence in the human pathogen Vibrio vulnificus. Infect Immun 75: 3282-3289.

Lopez, C.S., and Crosa, J.H. (2007) Characterization of ferric-anguibactin transport in Vibrio anguillarum. Biometals 20: 393-403.

Meibom, K.L., Blokesch, M., Dolganov, N.A., Wu, C.Y., and Schoolnik, G.K. (2005) Chitin induces natural competence in Vibrio cholerae. Science 310: 1824-1827.

Ninawe, A.S., and Selvin, J. (2009) Probiotics in shrimp aquaculture: avenues and challenges. Crit Rev Microbiol 35: 43-66.

Okada, K., lida, T., Kita-Tsukamoto, K., Honda, T. (2005) Vibrios commonly possess two chromosomes. J Bacteriol. 187:752-7.

Park, E.D., Lightner, D.V., and Park, D.L. (1994) Antimicrobials in shrimp aquaculture in the United States: regulatory status and safety concerns. Rev Environ Contam Toxicol 138: 1-20. Pritchard, L., White, J.A., Birch, P.R., and Toth, I.K. (2006) GenomeDiagram: a python package for the visualization of large-scale genomic data. Bioinformatics 22: 616-617.

Reynaud, Y., Saulnier, D., Mazel, D., Goarant, C., and Le Roux, F. (2008) Correlation between detection of a plasmid and high-level virulence of Vibrio nigripulchritudo, a pathogen of the shrimp Litopenaeus stylirostris. Appl Environ Microbiol 74: 3038-3047.

Rosenberg, E., and Falkovitz, L. (2004) The Vibrio shiloi/Oculina patagonica model system of coral bleaching. Annu Rev Microbiol 58: 143-159.

Sakai, T., Hirae, T., Yuasa, K., Kamaishi, T., Matsuyama, T., Miwa, S. et al. (2007) Mass mortality of cultured kuruma prawn Peneaus japonicus caused by Vibrio nigripulchritudo. Fish Pathology 42: 141-147.

Satchell, K.J. (2007) MARTX, multifunctional autoprocessing repeats-in-toxin toxins. Infect Immun 75: 5079-5084.

Saulnier, D., Avarre, J.C., Le Moullac, G., Ansquer, D., Levy, P., and Vonau, V. (2000) Rapid and sensitive PCR detection of Vibrio penaeicida, the putative etiological agent of syndrome 93 in New Caledonia. Dis Aquat Organ 40: 109-115.

Sheahan, K.L., and Satchell, K.J. (2007) Inactivation of small Rho GTPases by the multifunctional RTX toxin from Vibrio cholerae. Cell Microbiol 9: 1324-1335.

Sheahan, K.L., Cordero, C.L., and Satchell, K.J. (2007) Autoprocessing of the Vibrio cholerae RTX toxin by the cysteine protease domain. Embo J 26: 2552-2561. 
Thompson, F.L., lida, T., and Swings, J. (2004) Biodiversity of vibrios. Microbiol Mol Biol Rev 68: 403-431, table of contents.

Vallenet, D., Labarre, L., Rouy, Z., Barbe, V., Bocs, S., Cruveiller, S. et al. (2006) MaGe: a microbial genome annotation system supported by synteny results. Nucleic Acids Res 34: 53-65.

Winstanley, C. (2002) Spot the difference: applications of subtractive hybridisation to the study of bacterial pathogens. J Med Microbiol 51: 459-467.

Tables 
Table 1: PCR detection of pA1066 (PCR products are localized in Figure 1) within the strain collection.

\begin{tabular}{|c|c|c|c|c|c|c|c|c|c|c|c|}
\hline Virulence & Strain & 1 & 2 & 3 & 4 & 5 & 6 & 7 & 8 & 9 & 10 \\
\hline \multirow[t]{17}{*}{ HP } & SFn1 & + & + & + & + & + & + & + & + & + & + \\
\hline & POn19 & + & + & + & + & + & + & + & + & + & + \\
\hline & SFn27 & + & + & + & + & + & + & + & + & + & + \\
\hline & SFn135 & + & + & + & + & + & + & + & + & + & + \\
\hline & SFn127 & + & + & + & + & + & + & + & + & + & + \\
\hline & AgMn13 & + & + & + & + & + & + & + & + & + & + \\
\hline & SFn49 & + & + & + & + & + & + & + & + & + & + \\
\hline & AgMn7 & + & + & + & + & + & + & + & + & + & + \\
\hline & POn2 & + & + & + & + & + & + & + & + & + & + \\
\hline & SFn128 & + & + & + & + & + & + & + & + & + & + \\
\hline & AgMn10 & + & + & + & + & + & + & + & + & + & + \\
\hline & SFn106 & + & + & + & + & + & + & + & + & + & + \\
\hline & SFn2 & + & + & + & + & + & + & + & + & + & + \\
\hline & AgMn12 & + & + & + & + & + & + & + & + & + & + \\
\hline & SFn105 & + & + & + & + & + & + & + & + & + & + \\
\hline & AgMn8 & + & + & + & + & + & + & + & + & + & + \\
\hline & AgMn9 & + & + & + & + & + & + & + & + & + & + \\
\hline \multirow[t]{11}{*}{ MP } & BDn2 & + & + & + & + & + & + & - & - & + & + \\
\hline & BDn1 & + & + & + & + & + & + & - & - & + & + \\
\hline & BLFn1 & + & + & + & + & + & + & - & - & + & + \\
\hline & BLFn2 & + & + & + & + & + & + & - & - & + & + \\
\hline & Wn13 & + & + & + & + & + & + & - & - & + & + \\
\hline & ENn2 & + & + & + & + & + & + & - & - & + & + \\
\hline & AQn1 & + & + & + & + & + & + & - & - & + & + \\
\hline & Wn1 & + & + & + & + & + & + & - & - & + & + \\
\hline & Fn1 & + & + & + & + & + & + & - & - & + & + \\
\hline & MT1 & + & + & + & + & + & + & - & - & + & + \\
\hline & AQn2 & + & + & + & + & + & + & - & - & + & + \\
\hline \multirow[t]{15}{*}{ NP } & FTn1 & - & - & + & - & - & + & + & + & - & - \\
\hline & POn13 & - & - & + & - & - & + & - & - & - & - \\
\hline & SOn1 & - & - & - & - & - & + & - & - & - & - \\
\hline & AgMn1 & - & - & - & - & - & + & - & - & - & - \\
\hline & SOn2 & - & - & - & - & - & - & - & - & - & - \\
\hline & ESn2 & - & - & - & - & - & - & - & - & - & - \\
\hline & ENn1 & - & - & - & - & - & - & - & - & - & - \\
\hline & POn10 & - & - & - & - & - & - & - & - & - & - \\
\hline & Fn2 & - & - & - & - & - & - & - & - & - & - \\
\hline & SFn115 & - & - & - & - & - & - & - & - & - & - \\
\hline & SVn3 & - & - & - & - & - & - & - & - & - & - \\
\hline & AgMn3 & - & - & - & - & - & - & - & - & - & - \\
\hline & POn12 & - & - & - & - & - & - & - & - & - & - \\
\hline & POn4 & - & - & - & - & - & - & - & - & - & - \\
\hline & SFn118 & - & - & - & - & - & - & - & - & - & - \\
\hline
\end{tabular}


Table 2

Strains used and constructed in this study

\begin{tabular}{|l|l|}
\hline Name & Plasmid(s) \\
\hline SFn1 & pB1067, pA1066 \\
\hline VN110 & pSW110 \\
\hline VN157 & pA1066 \\
\hline VN68 & pB1067::pSW68 \\
\hline VN120 & pB1067::pSW120 \\
\hline
\end{tabular}

Table 3

Plasmids used and constructed in this study

\begin{tabular}{|l|l|l|}
\hline Plasmid & Description & Reference \\
\hline pSW25T & oriV $_{\mathrm{R} 6 \mathrm{~K} \gamma} ;$ oriT $_{\mathrm{RP} 4} ;\left[\mathrm{Spec}^{\mathrm{R}}\right]$ & Demarre et al., 2005 \\
\hline pSW110 & pSW25T:: $(2700-3400)_{\mathrm{pB} 1067}$ & This study \\
\hline pSW68 & pSW25T:: (2000-2530 $)_{\mathrm{pB} 1067}$ & This study \\
\hline pSW120 & pSW25T:: (28-480) ${ }_{\mathrm{pB} 1067}$ & This study \\
\hline
\end{tabular}

\section{Figures}




\section{Figure 1}

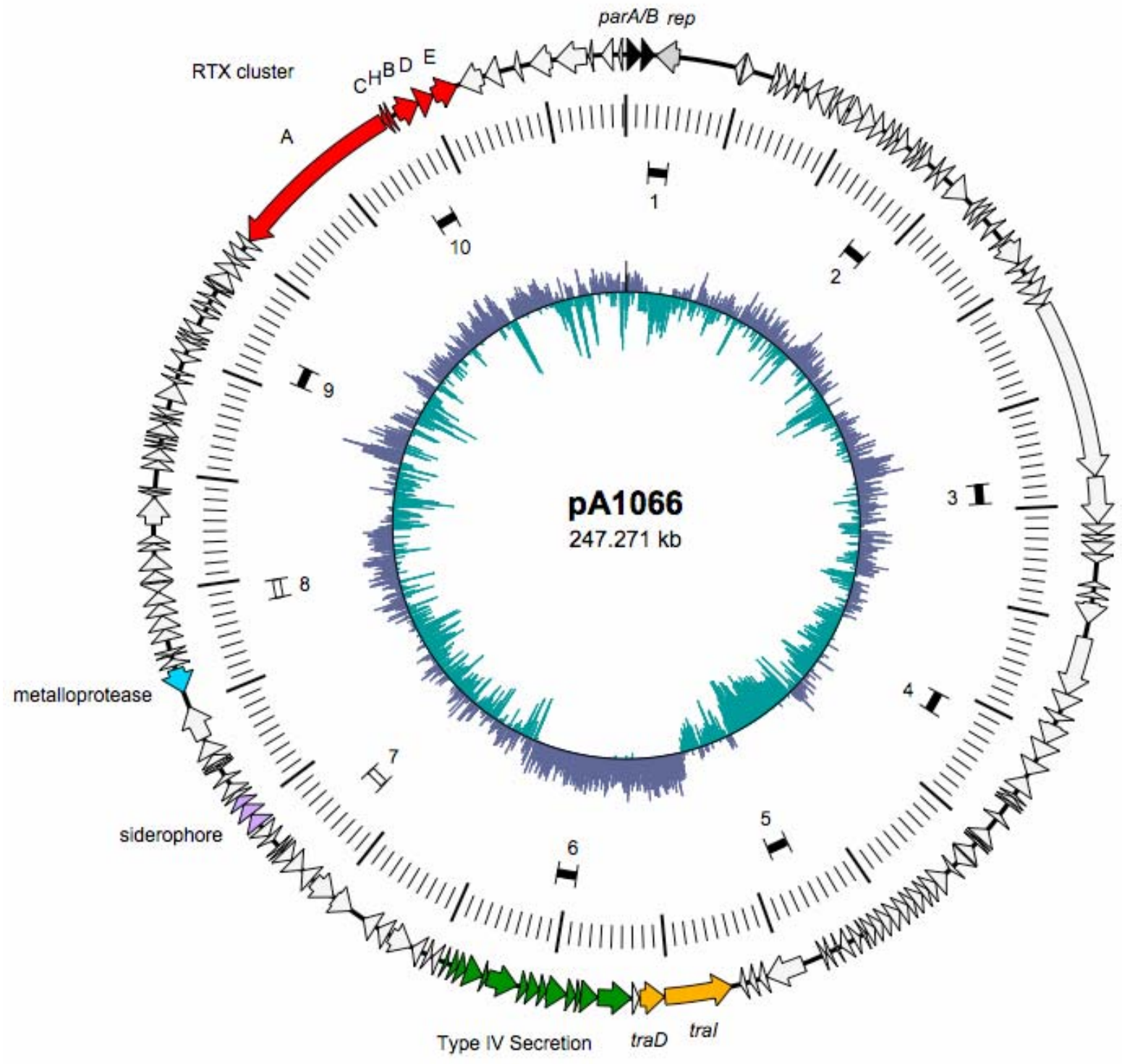

\section{Figure 1}

Circular representation of the V. nigripulchritudo pA1066 plasmid. From the outside inwards: the $1^{\text {st }}$ circle shows pA1066 ORFs (in yellow replication and partitioning; in green and orange conjugative transfer; in red MARTX cluster; in blue metalloprotease; in purple siderophore $A B C$ transporter ); the $2^{\text {nd }}$ circle shows the scale (small tick marks $=1 \mathrm{~kb}$; large tick marks $=10$ $\mathrm{kb})$; the $3^{\text {rd }}$ circle shows the PCR products amplified for pA1066 detection and genotyping; $4^{\text {th }}$ circle shows the GC percent. 
Figure 2

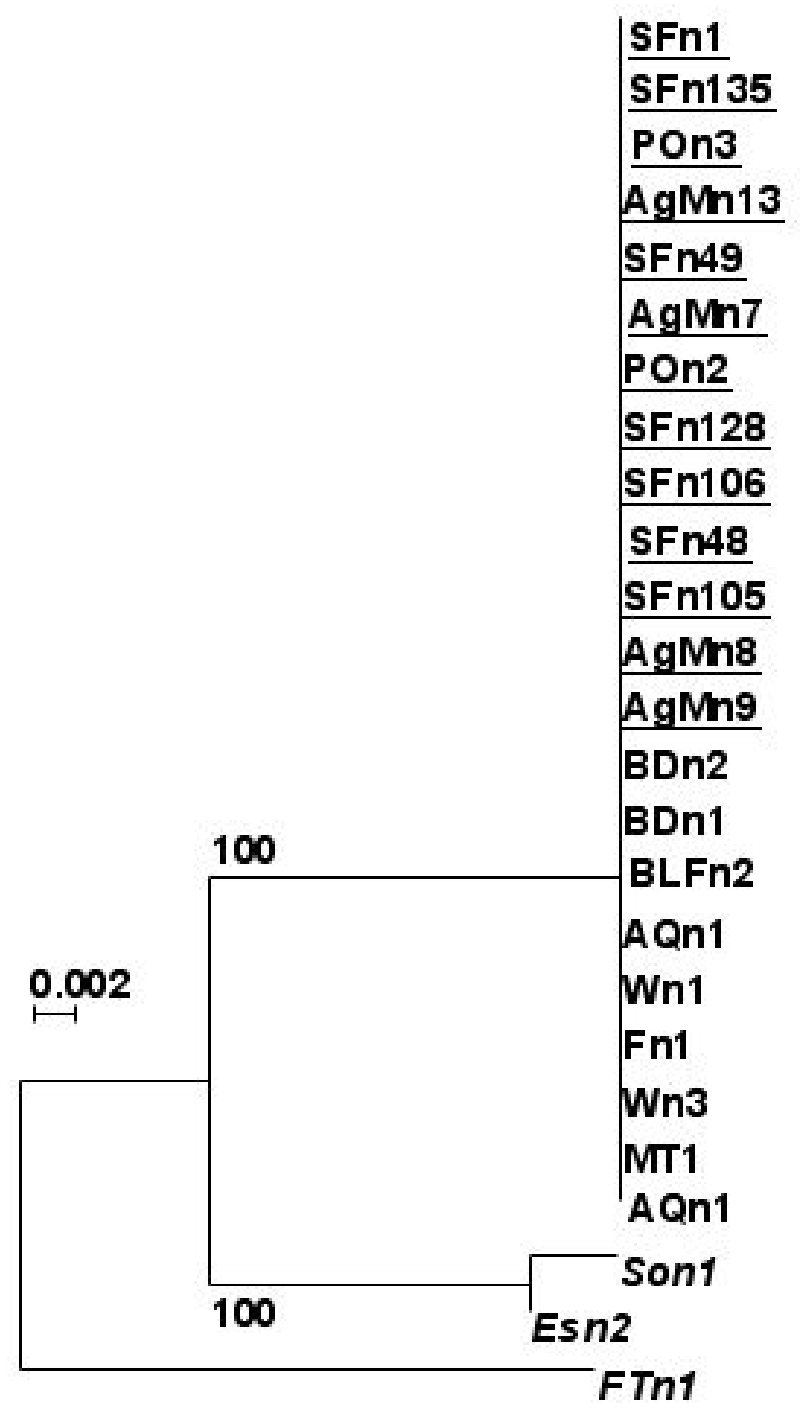

Figure 2: Phylogenetic tree of the pA1066 rep gene. The tree was built by the Neighborjoining method based on sequences aligned using Seaview. Branch lengths are drawn to scale and are proportional to the number of nucleotide changes. Number at each node represents the percentage value given by bootstrap analysis of 1000 replicates. HP strains are in bold and underline, MP in bold, NP in italic. 
Figure 3
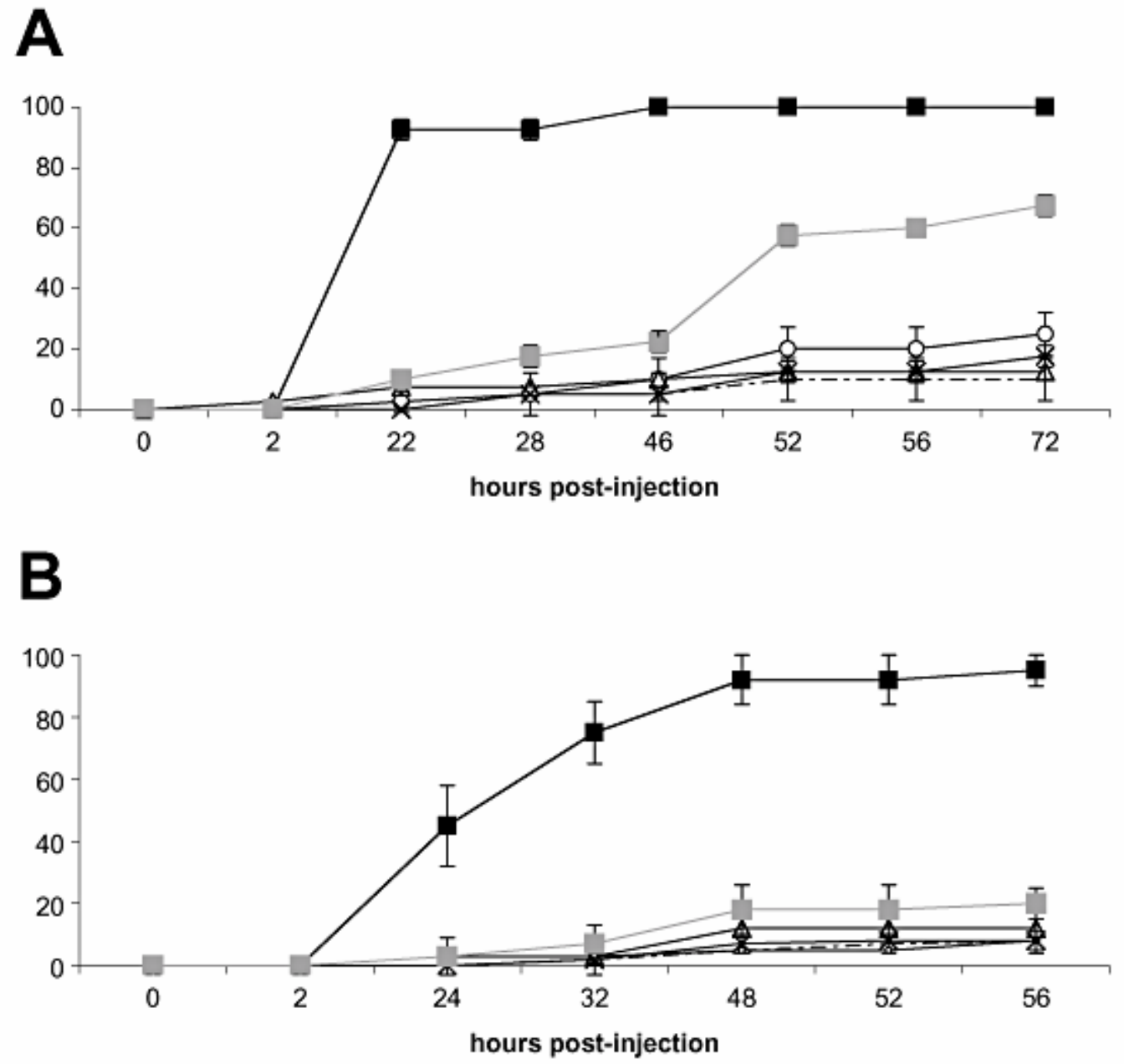

\section{Control}

SFn1 (pA1066+pB1067)

VN68 (pB1067::pSW68) $\longrightarrow$ VN110 (pSW110)

$\neg-$ VN120 (pB1067::pSW120)

VN157 (pA1066)

Figure 3: Shrimp mortality in response to experimental infection with $V$. nigripulchritudo. For the injection challenge (A), 50 to $500 \mathrm{CFU}$ of the tested strain was intramuscularly injected into shrimp ( $n=10$ to 20 , in duplicate). For the immersion challenge (B), shrimp ( $n=10$ to 20 , in triplicate) were incubated 2 hours with $10^{5} \mathrm{CFU} / \mathrm{mL}$ of $V$. nigripulchritudo, then transferred to $100 \mathrm{~L}$ tanks containing filtrated seawater. Survival was monitored daily over a 3-day period. 
Figure 4

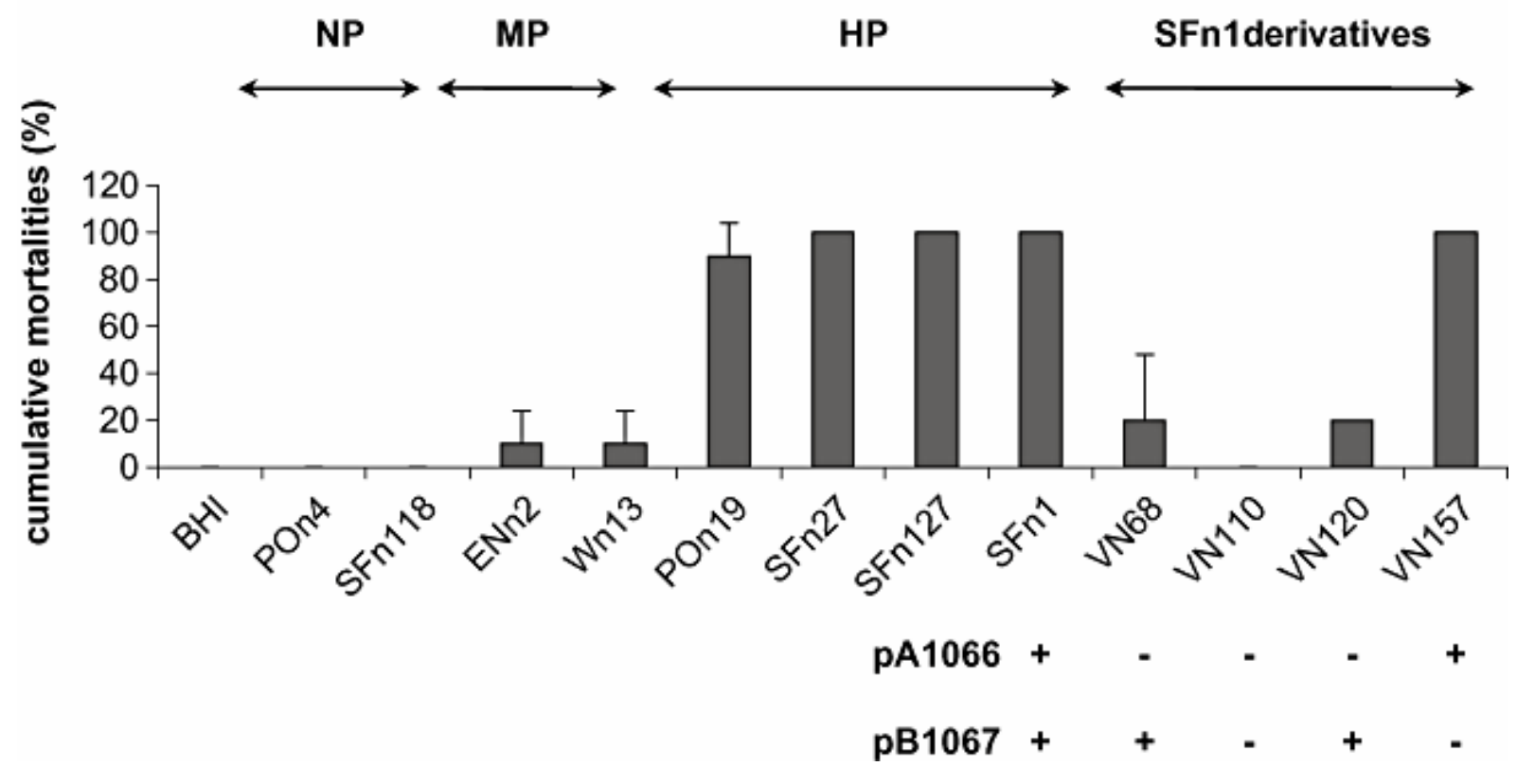

Figure 4: Shrimp mortality in response to injection of $V$. nigripulchritudo culture supernatants. Animals ( $\mathrm{n}=8$ to 12) were injected with $100 \mu \mathrm{L}$ of bacterial supernatant prepared from overnight cultures. Survival was assessed after 24 hours. Experiments were conducted in duplicate. 


\section{Supplementary data}

Table 1

Oligonucleotides used in this study

\begin{tabular}{|c|c|}
\hline Oligonucleotide & Sequence (5'-3') \\
\hline SW25seqS & CGGTATCGATAAGCTTGATATC \\
\hline SW25seqAS & GGCGGCCGCTCTAGAACTAGT \\
\hline pB2700f & GCCCGGATCCCGCGCTATCGCTTGTCGGCC \\
\hline pB3400r & GCCCGGATCCAAGAGCAGGGCTTTATATAGGACG \\
\hline pB2000f & GCCCGGATCCGGACAGGATTTAACATTGAGGGG \\
\hline pB2530r & GCCCGGATCCGAAAGGGCACCCAATAGGGTGC \\
\hline pB28f & GCCCGGATCCTTGTTCGTGTTGAGAATAGC \\
\hline pB480r & GCCCGGATCCCAGCATTTAAGGCCTTACTC \\
\hline pA1f & TTGGCTATCATTGCTGGTGCA \\
\hline pA1r & TGTCTTCGATGGGACTGAACAA \\
\hline pA2f & ATGCAGAAGCCTGGCGGGA \\
\hline pA2r & GGAGCAGAGACATTAAGTCGGT \\
\hline pA3f & TCCCGTGTCCCATGCGTTCCAT \\
\hline pA3r & ACGCAAACGCAGAAGGCAATGT \\
\hline pA4f & CGTAGGGTTCCTCAGAGAATTCT \\
\hline pA4r & GCATTGCTCCCATATTCGCAGG \\
\hline pA5f & GGCAGGTTGGTCAAAGCGGTCT \\
\hline pA5r & TGAGTAAGCAGTGGCAGCGTAC \\
\hline pA6f & GTCTCGGGTTTCTTCTGGTTCA \\
\hline pA6r & TGGAACGACGATGAACTCGCCT \\
\hline pA7f & CTAACAAGCCAGCTCCAGGT \\
\hline pA7r & ACCAAAGTCAGACAATCCTCTG \\
\hline
\end{tabular}




\begin{tabular}{|l|l|}
\hline pA8f & AGGACGTTCTGGTGGTGGGCTA \\
\hline pA8r & CTGAAACAATGCGTAGCGATAG \\
\hline pA9f & CTTTGAGTCCCATAACCCCCGT \\
\hline pA9r & TCATAGAATCGTTCCCACTGGT \\
\hline pA10f & CAACACGGGAGCTGATGCTGT \\
\hline pA10r & CATAGTCTGATTATTGAGCGT \\
\hline
\end{tabular}

Figure 1S

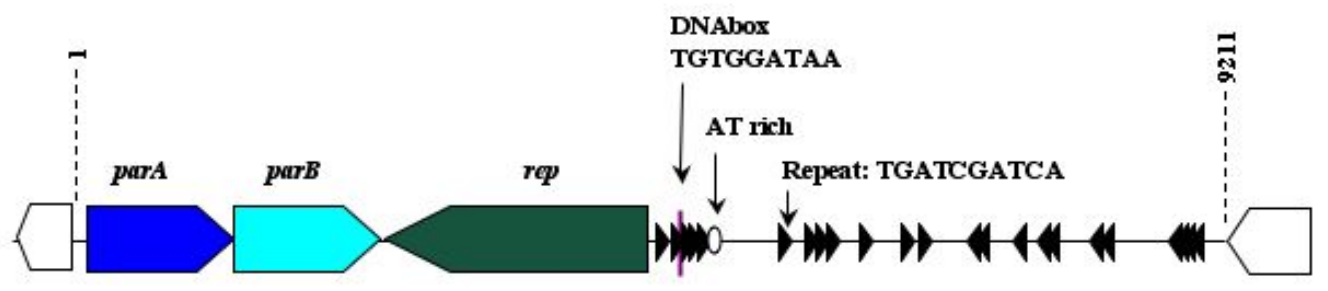

The replication/segregation module. 


\section{Figure 2S}

A

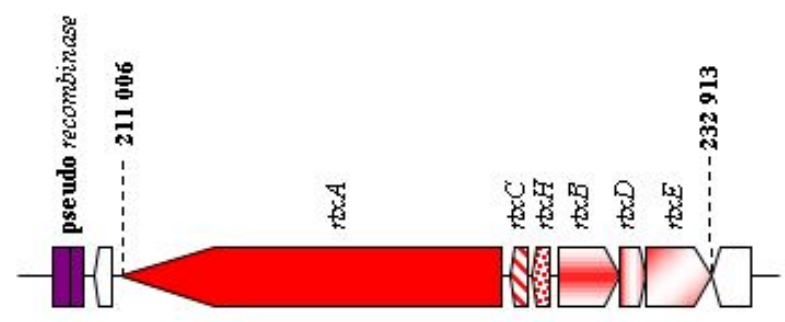

B

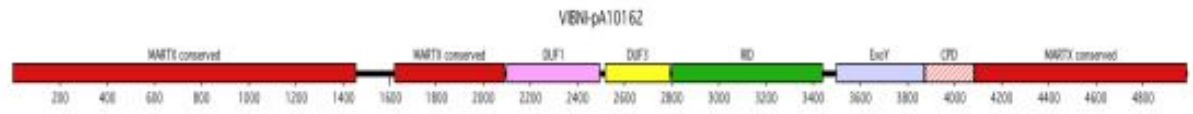

1-2093: MARTX conserved $=$ repeat regions found in all MARTX toxin 1461-1619: Gap in alignment also found in P. luminescens toxin (Pl1) 2097-2498: DUF1 found in VcRC385 and $Y$, enterocolitica 2517-2796: DUF3 found in $V$, vulnificus and Xenorhabdus sp 2797:3441: RID (rho inactivation domain) characterized in $V$. cholerae 3497:3441: ExoY (adenylate cyclase) found in VcRC385 3873:4085: CPD (cystein protease domain) found in all MARTX 4086:4990: MARTX conserved = repeat regions found in all MARTX toxins

The MARTX (multifunctional autoprocessing repeats in toxin) gene cluster $(A)$ and domain annotation (B). 
Figure 3S

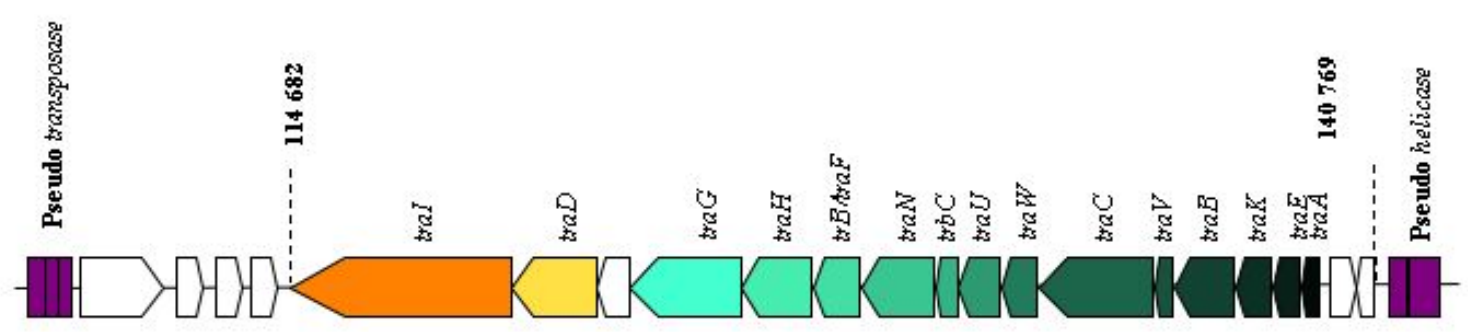

The putative conjugative machinery. This cluster includes homologs of the core proteins of a Type IV secretion system (in green), the coupling protein TraD and a relaxase-helicase Tral (yellow and orange). 